\title{
A Visually Guided Framework for Lung Segmentation and Visualization in Chest CT Images
}

\author{
Shouren Lan ${ }^{\mathrm{a}}$, Xin Liu ${ }^{\mathrm{b}}$, Lisheng Wang ${ }^{\mathrm{a}}$, Chaoyi Cui ${ }^{\mathrm{c}, *}$ \\ ${ }^{a}$ Institute of Image Processing and Pattern Recognition, Department of Automation, Shanghai Jiao Tong University, Shanghai, 200240, P. R. China. \\ ${ }^{b}$ Center for Machine Vision and Signal Analysis, University of Oulu, Finland. \\ ${ }^{c}$ Department of Vascular Surgery, Shanghai Ninth People's Hospital, Shanghai Jiao Tong University School of Medicine, Shanghai, 200011, P. R. China.
}

\begin{abstract}
Lung cancer is the leading cause of cancer-related death worldwide and this also stimulates the development of various computeraided diagnosis (CAD) systems. But the conventional lung segmentation methods can't satisfy the needs of the clinicians in lung cancer diagnosis and surgery. It is very important to provide a segmentation and visualization framework for the clinicians instead of radiologists in outpatient service. Therefore we propose a visually guided method based on a 2D feature space and spatial connectivity computation to reduce the dependence on the radiologists for lung segmentation and visualization. Our framework consists of three main processing steps. Firstly, a 2D feature space of CT scalar versus gradient magnitude is constructed. Secondly, the attribute distribution region of the lungs is selected in the $2 \mathrm{D}$ feature space, and then the lungs are extracted from the determined voxels by spatial connectivity computation. Finally, the lungs and pulmonary nodules are visualized simultaneously with different colors and opacities in volume rendering. Experimental results show that the proposed framework is efficient for outpatient service and can provide an intuitive segmentation process and nodules information.
\end{abstract}

Keywords: Lung Segmentation, 2D Feature Space, Spatial Connectivity, Volume Rendering, Visually Guided

\section{Introduction}

Lung cancer is the leading cause of cancer-related death worldwide. In CT lung cancer screening, a large number of CT scans have to be analyzed, which is an encumbrance for radiologists. In addition, the information they provide can not well satisfy the needs of the clinicians. Therefore, a fast lung segmentation and visualization framework that clinicians want will be important for clinicians in outpatient service. This makes them independent of radiologist. Hence, a fast and intuitive lung segmentation and visualization method could have remarkable practical significance and clinical value for optimize screening efficiency. Thus far, many lung segmentation methods have been studied by researchers, see $[1,2,3,4,5,6,7,8,9,10,11$, $12,13,14,15,16,17]$ and references therein. Here, we classify them simply into two classes: the automatic segmentation methods and the interactive segmentation methods.

The automatic segmentation methods include mainly the threshold methods, priori knowledge methods and machine learning methods. The threshold method $[2,3,4]$ is the simplest and most conventional method, but it can't separate the left and right lungs without post-processing. Pu et al. [5] proposed an adaptive border marching algorithm for fully automatic lung segmentation in 2D sectional slice. Sun et al. [6] presented a robust active shape model matching method. However, these methods need to establish a complete shape dictionary model.

\footnotetext{
${ }^{*}$ Corresponding author.

Email address: cuichoi8432@163.com (Chaoyi Cui )
}

Noor et al. [7] proposed an automatic segmentation method based on morphology and texture paradigm that segregate normal and diseased lungs. Mansoor et al. [8] combined fuzzy connectedness with a glossary of abnormal CT imaging patterns to automatically segment pathological lungs. However, excessive computation time and the glossary limit its application. The atlas-based method [9] was also studied to segment lungs, but due to anatomical variability, it's not available. Moreover, the required computation time is long because of the registration process. Recently, the machine learning methods have also been studied by many researchers. For example, the SVM method $[10,11]$ combines texture information with local shape to generate a classifier for lung segmentation. Ceylan et al. [12] presented a complex-valued artificial neural network method with complex wavelet transform for lung segmentation. In these machine learning methods, the most challenging task is the selection of feature sets appropriate for different training data. In addition, they are difficult to use for domain experts such as physicians.

Currently, the interactive segmentation methods have been developed because of the complex and time consuming nature of the automatic segmentation methods. The interactive methods include region growing methods [13, 14, 15], boundary tracking methods $[16,17]$ and graph cuts methods $[19,20,21,22]$. The region growing method is widely used in lung segmentation, but in many cases designing an appropriate rule to stop the growth of the region without causing over-segmentation is a complex task. Although the edge tracing methods can simplify threshold selection, but also face 
the problem of stop rules in tracing process. The graph-cuts method is based on graph theory [18] and it has been studied by many researchers as a new image segmentation method which could have intuitive user interactions. Sun et al.[19] proposed a graph-based approach for simultaneous segmentation of lungs in 4D CT scans with an expert-guided computer-aided refinement, but the user interactions in this method are complex and time-consuming. Nakagomi et al. [20] proposed a novel graph cuts algorithm by incorporating multiple shapes and prior information on neighbor structures and obtained high accuracy in segmentation. Ali et al. [21] used graph cuts for unsupervised segmentation of the lungs from low-dose CT scans images. Dai et al. [22] proposed an improved graph cuts algorithm with Gaussian mixture models. All these approaches applied the graph cuts theory to lung segmentation to obtain high accuracy and also to improve user interactions for the domain experts. However, these approaches are time-consuming, which is problematic for outpatient service.

As discussed above, the automatic lung segmentation methods usually have low usability for domain experts due to require excessive processing time. The conventional interaction methods have poor interactions or interfaces, and usually high computation time; even the fastest method based on graph cuts [19] needs approximately 4.3 minutes of interaction time. In addition, these two kinds of methods can't provide visualization results of pulmonary nodules, together with the lungs. In order to provide a more intuitive method, while avoiding the high computation time of the above mentioned methods, this paper intends to present a visually guided framework to fast segment the left and right lungs and visualize the pulmonary nodules in volume rendering. This framework consists of three main processing steps. The first step is to select a region of attributes distribution of the lungs in 2D feature space. The second step is to visually extract the lungs by spatial connectivity computation. The third step is to visualize the lungs and pulmonary nodules simultaneously with different colors and opacity. The framework provides a good guarantee in the outpatient service for clinicians, especially in face of more and more patients with pulmonology. Therefore, the clinicians are not limited to the results of what the radiologist provided in the diagnostic process.

The rest of the paper is organized as follows. Section 2 describes the framework for segmenting and visualizing the lungs by combining the 2D feature space and spatial connectivity. Section 3 provides many experiments. Section 4 presents an evaluation of the method in terms of its accuracy and efficiency. Section 5 summarizes our contributions and conclusions

\section{Methods}

In order to provide a segmentation and visualization framework for the clinicians instead of radiologists in outpatient service, we propose a visually guided framework to reduce the dependence on the radiologists for lung segmentation and visualization. The flowchart of the framework is shown in Fig.1. Below, we will introduce each step for lung segmentation and visualization in detail.

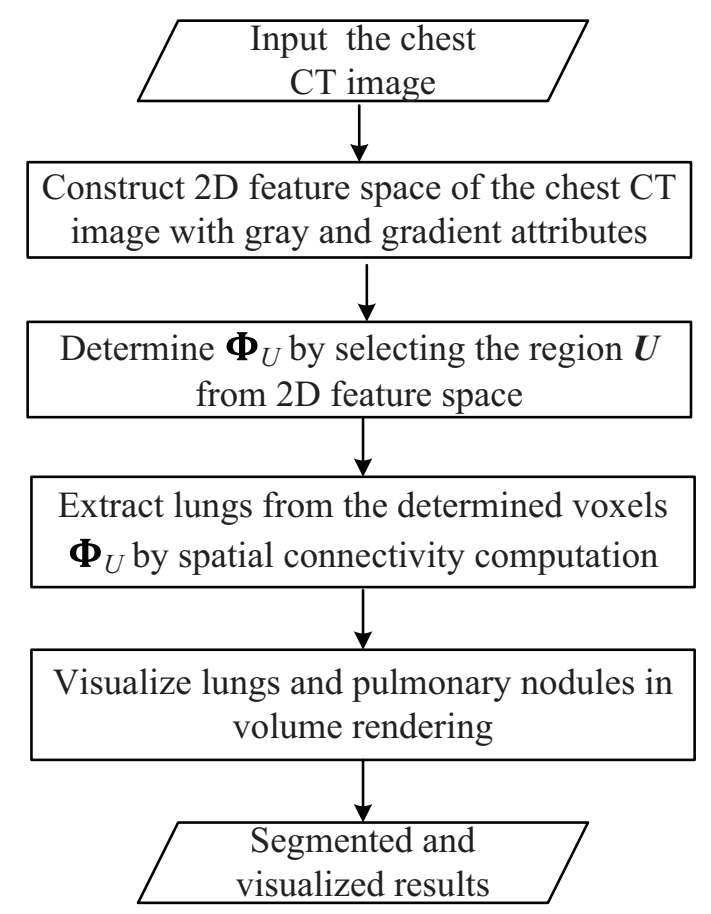

Figure 1: The flowchart of the framework.

\subsection{Constructing and Analyzing the $2 D$ feature space}

When viewed on sectional CT slices, the anterior junctions between the left and right lungs may be very thin with high magnitude of gradient, as shown in Figs.2a. In many cases, gray-scale thresholding fails to separate the left and right lungs near these junctions [1, 3], as shown in Figs.2b. Therefore, we joined in gradient attributes on the basis of the gray to construct the 2D feature space. The 2D feature space based on scalar value and gradient magnitude for visualization classification is widely used in volume rendering [24]. However, lung segmentation with $2 \mathrm{D}$ feature space is a novel approach in medical image segmentation. The $2 \mathrm{D}$ feature space can be defined as a 2D histogram which describes and counts the attribute distribution of the gray and gradient value in the chest CT image. For scalar data, the gradient is the first derivative measure. The gradient magnitude is a scalar quantity which describes the local rate of change in the scalar field. For notational convenience, this paper uses $\|\nabla f(x, y, z)\|$ to indicate the magnitude of the gradient of $f(x, y, z)$, where $f(x, y, z)$ is the scalar function representing the chest CT image.

Here, 10 2D feature spaces can be constructed from 10 different chest CT images, as shown in Fig.3. The horizontal coordinate represents the gray scale, and the vertical coordinate represents the magnitude of gradient in the $2 \mathrm{D}$ feature space. These 2D feature spaces have very similar histogram distribution. In fact, the attribute distribution of all chest CT images is an arched shape in the 2D feature space. The consistency distribution of different chest CT images can provide a unified rule in the choice of gray and gradient for lung segmentation in 2D feature space.

Here, the arched distribution was divided into three parts: 


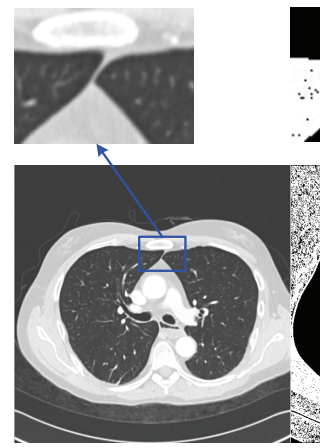

(a)
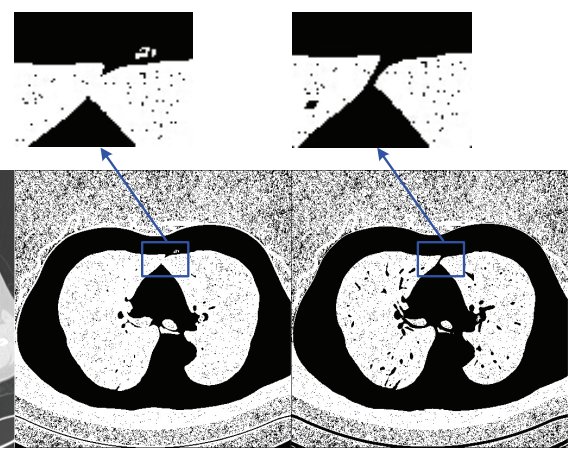

(b)

(c)
Figure 2: (a) Original CT slice. (b) Segmented result using gray-scale thresholding. (c) Segmented result using 2D feature space.

the left end of the arch (denoted by $R 1$ represents the homogeneous regions of low gray gradient), the middle section of the arch (denoted by $R 2$ represents inhomogeneous regions of high gradient) and the right end of the arch (denoted by $R 3$ represents homogeneous regions of high gray and low gradient), as shown in Fig.4a. According to the statistic of Fig.4b showed that the lungs corresponds to region R1, the soft tissues and bones corresponds to region $\mathrm{R} 3$, the small vascular structure and skin, as well as the outer edge of the lungs corresponds to region R2 in $2 \mathrm{D}$ feature space. The statistic are further demonstrated in Fig.4c. In addition, we also visualize the voxels of the three divided regions determined in volume rendering; see Fig.4d, $4 \mathrm{e}$ and $4 \mathrm{f}$. In the following, we will introduce the application of the $2 \mathrm{D}$ feature space and spatial connectivity to separate and visualize the lungs in the chest $\mathrm{CT}$ images.

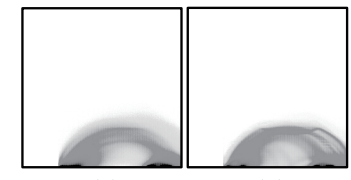

(a)

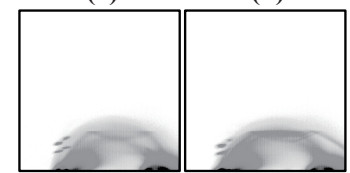

(f) (g)

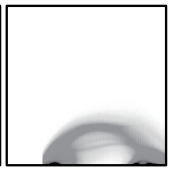

(c)

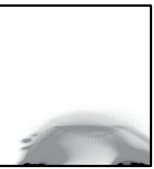

(h)

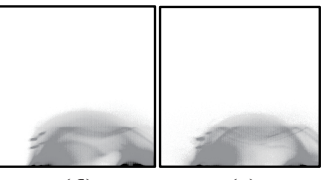

(d)
Figure 3: The 2D feature space is a log-scale joint histogram of scalar value versus gradient magnitude. Dark regions represent large numbers of data value occurrences.

\subsection{The visually guided framework for extracting and visual- izing the lungs and pulmonary nodules}

In this section, we will introduce a visually guided framework for separating and visualizing the lungs with pulmonary nodules in the chest $\mathrm{CT}$ images by combining the $2 \mathrm{D}$ feature space and spatial connectivity. This framework includes primarily region selection in the $2 \mathrm{D}$ feature space, visual extraction of the lungs by spatial connectivity computation and visualization of the lungs and nodules in volume rendering.

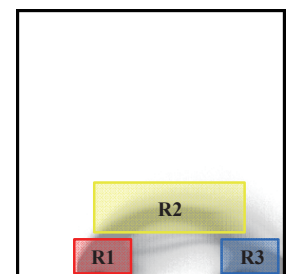

(a)

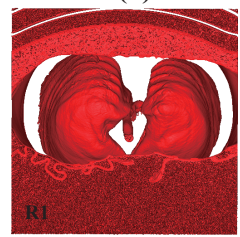

(d)

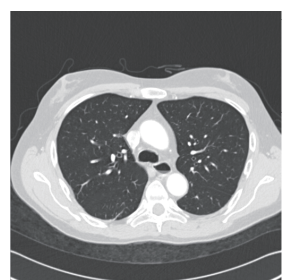

(b)

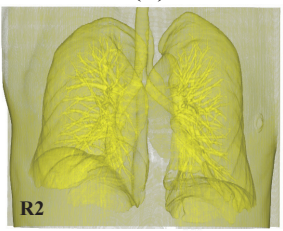

(e)

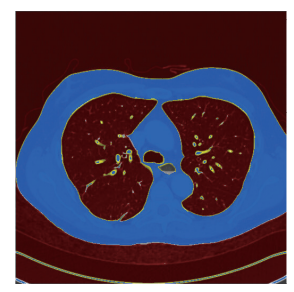

(c)

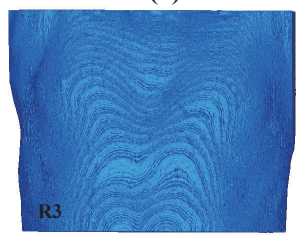

(f)
Figure 4: (a) Three divided regions in 2D feature space. (b) Original CT slice. (c) Mapping the divided regions to sectional CT slices with pseudo-color. (d)(f) visualization of mapping results.

\subsubsection{Selecting region $R I$ in the $2 D$ feature space}

In Section 2.1 of this paper we discussed the attributes distribution of the chest CT images and determined the distribution region of the lungs in the $2 \mathrm{D}$ feature space (Region $R 1$ in Fig.4a). Now, we only need to select region $R 1$ to obtain the voxel set of the lungs, as shown in Fig.5a. When selecting a region $U$ (this paper assumes $U=R 1$, representing the selected gray and gradient interval) from the $2 \mathrm{D}$ feature space of a chest CT image $f(x, y, z)$ for the lungs, a set of voxels (denoted by $\Phi_{U}$, Eq. 1$)$ is determined from $f(x, y, z)$. Although $\Phi_{U}$ also contains some uninteresting structures, they are not connected spatially, as shown in Fig.2c and Fig.5b. Based on this property, we can easily extract the lungs from $\Phi_{U}$ by spatial connectivity computation.

$$
\Phi_{U}=\{(x, y, z):(f(x, y, z),\|\nabla f(x, y, z)\|) \in U\}
$$

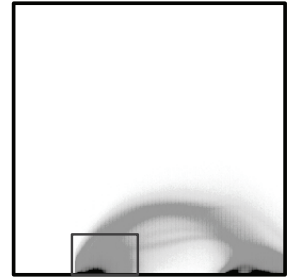

(a)

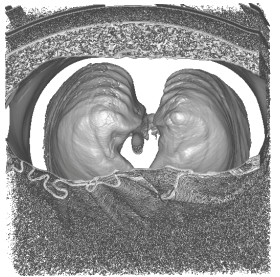

(b)

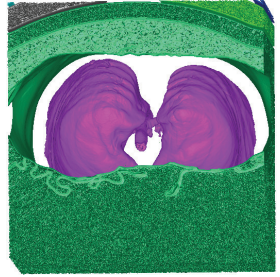

(c)
Figure 5: (a) Select region $U$ in 2D feature space and determine $\Phi_{U}$. (b) Visualize all voxels of $\Phi_{U}$ in volume rendering. (c) Visualize all connected sets of $\Phi_{U}$ with different colors after spatial connectivity computation.

\subsubsection{Extracting the lungs from $\Phi_{U}$ using the spatial connec- tivity}

Each structure is a spatially connected structure in the chest CT images. When a suitable region $U$ is selected from the 2D feature space, each structure formed by numerous structure voxels in $\Phi_{U}$ will retain the connectivity as well. Furthermore, 
different structures form different connected sets in $\Phi_{U}$. These different connected sets of voxels can be extracted from $\Phi_{U}$ by the following tracing algorithm:

Step 1 Mark all voxels in $\Phi_{U}$ as not-visited ones.

Step 2 Perform the following operations (Step 2.1-Step 2.2) circularly until all voxels in $\Phi_{U}$ are marked as the voxels visited.

Step 2.1 Select a not-visited voxel from $\Phi_{U}$ as a seed, mark it as a visited voxel and push it into a stack denoted by $H$.

Step 2.2 Perform the following operation (Steps 2.2.12.2.2) circularly until $H$ is empty:

Step 2.2.1 Pop up a voxel from $H$.

Step 2.2.2 Consider all voxels in the 18 - neighborhood of the voxel in the following way. If a voxel is not in $\Phi_{U}$ or is visited before, then skip it. If a voxel is in $\Phi_{U}$ and not visited before, then push it into $H$, and mark it as the visited voxel.

Finally, all connected sets in $\Phi_{U}$ are extracted with different colors, as shown in Fig.5c. Nevertheless, we are interested only in the connected set of the lungs, instead of all connected sets in $\Phi_{U}$. To extract all connected sets also cost remarkably more time. So it is necessary to specify the seed point interactively at Step 2.1 of the tracing algorithm to extract the lungs. The algorithm will be terminated when the lungs are extracted. It is important that the framework provides an intuitive and efficient seed selection method in volume rendering results instead of the 2D sectional slice.

Interactive 3D medical image cutting is widely used as a flexible manual segmentation tool to extract regions of interest in 3D CT images [23]. Similarly, it has also been modified as a seed selection tool for selecting the seed points in volume rendering result of $\Phi_{U}$ in this framework. This principle functions as a transformation between the object and screen coordinate frames, and it can be used to project voxels of 3D CT data onto the screen frame. That is, we can select a seed point on any position of the lungs in the volume rendering result and extract the lungs, as shown in Figs.6a and 6c. If we need to separate the left and right lungs, and we only need to select two seed points on the left and right bronchus, as shown in Figs.6b and $6 \mathrm{~d}$. To selectively extract the connected set requires very small amount of time, resulting in a clinically more acceptable processing time. Here, all operations are based on the visual results in volume rendering, and thus provide a fast and intuitive framework for lungs segmentation.

\subsubsection{Visualizing the lungs and pulmonary nodules in volume rendering}

Volume rendering $[26,27]$ is important visualization technique for exploring and visualizing structures in 3D images. By observing volume rendering results, users can discover some structures of interest and have intuitive understandings of their visual properties, such as shapes, spatial positions and spatial relations with other adjacent structures. This technique is very meaningful to the general clinicians in diagnosis of pulmonary diseases, because the lungs with pulmonary nodules can be visualized intuitively, especially in the bronchoscopy, needle biopsy procedure, and pulmonary segmentectomy. But the clinicians can't get the satisfactory visualization results from the

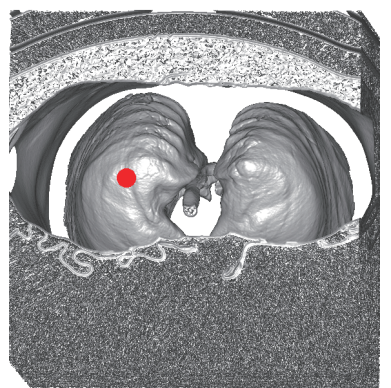

$\downarrow$ (a)

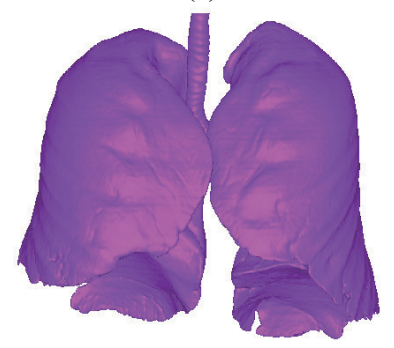

(c)

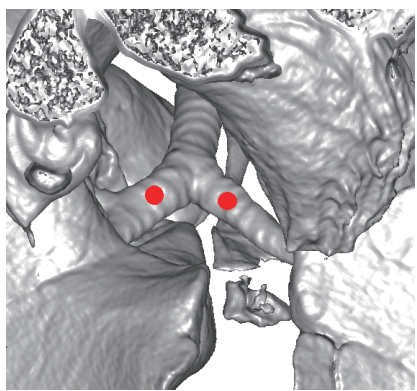

$\downarrow$ (b)

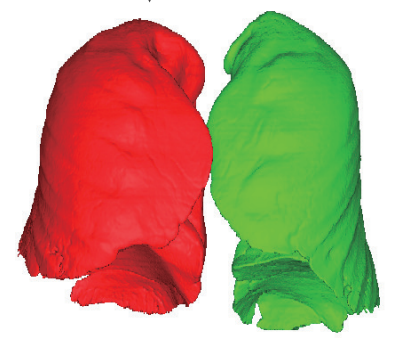

(d)
Figure 6: Seed points selection in volume rendering. (a) Select a seed point on any position of the lungs. (b) Select two seed points on the left and right bronchus. (c)-(d) Different extraction results from the selected seed points in (a)-(b).

radiologists, who provided the static volume rendering results by screenshot. Now we can provide a visualization module for the clinicians in outpatient service. The radiologists only need to provide the CT image and nodules information for the clinicians, who can segment and visualize the lungs and nodules with different colors and opacities in their own computers at any time. In addition, the clinicians can also segment the pulmonary nodules by region growing method for visualization without waiting for radiologist report. Fig.7 list three different chest CT image with nodules which distribute in different positions in chest CT images, see Fig.7a, 7b and 7c. We can find that it's not intuitive to observe the nodules locations and shapes based on sectional CT slices. Here, the proposed method provided an intuitive display of the nodules in volume rendering. By assigning low opacity values to the lungs, the occlusion of the lungs to the nodules can be avoided. Consequently, the clinicians can easily observe the positions of the pulmonary nodules by rotating the visualization results in their own computers, as shown in Fig.7d, 7e and 7f. This system is very good for the liberation of the clinician's dependence on radiologists.

\subsection{Repairing missing structures by a hole filling filter}

In this paper, we only select the region $R 1$ in $2 \mathrm{D}$ feature space to exclude high gradient region of the anterior junctions between the left and right lungs for separating the left and right lungs, as shown in Fig.2c. However, most areas of small vascular structure have high gray, which are not included in selected region R1 may be lost. Therefore pulmonary nodules having high gray may also be lost. Besides, these small vascular structures and pulmonary nodules both contain small gradient magnitude (inside of structure) that also contain large gradient mag- 


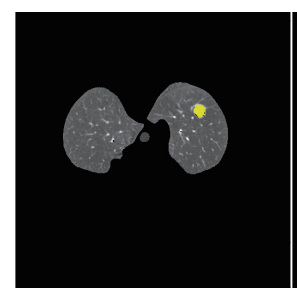

(a)

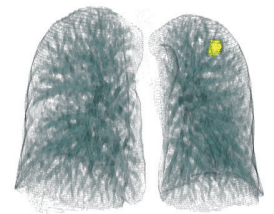

(d)

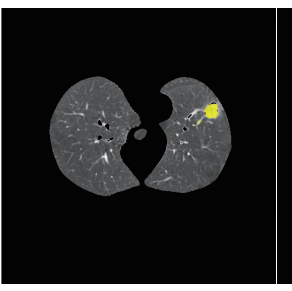

(b)

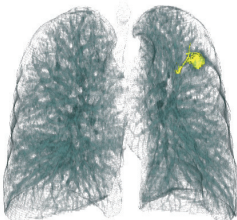

(e)

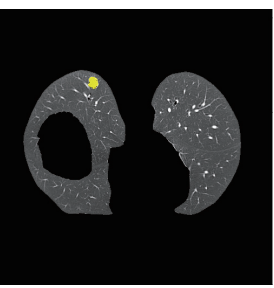

(c)

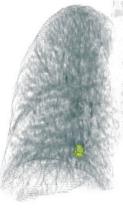

(f)
Figure 7: (a)-(c) Lung segmentation results, in which the pulmonary nodules are segmented by region growing with yellow color. (d)-(f) The segmented lungs and pulmonary nodules are visualized simultaneously with different colors and opacities in volume rendering.

nitude (boundary of structure). Therefore, these missing areas are difficult to be repaired by simply selecting the region $\mathrm{R} 2$ or R3. Here, we used a hole filling filter [25] to repair the pulmonary nodules and small vascular structures from the determined areas by region R1 and avoid false diagnosis. This filter can convert background pixels into the foreground only when the number of foreground pixels is a majority of the neighbors. The size of the neighborhood is defined along every dimension of the voxel, and the value on each dimension is used as the semi-size of a rectangular box. The hole filling filter can also be used in an iterative way, by calling it repeatedly either until no pixel changes or for a fixed numbers of iteration. In this paper, the size of the neighborhood was set to 2 , the neighborhood had the size $5 \times 5 \times 5$, and the majority value was set to 40 and the iteration number to 7 .

Fig.8 illustrates the effectiveness of the hole filling filter on a chest CT image. Fig. 8 a is the binary results from the spatial connectivity computation and Fig. $8 \mathrm{~b}$ is the segmentation result with the binary mask. We can find that the proposed method lost some structures of high gray gradient. These structures can be repaired after applying the hole filling filter operation, as shown in Figs.8c and 8d. The clinicians can also mark the pulmonary nodules in the repaired lung as a supplement to the radiologist's results, and then visualize the lung and pulmonary nodules in volume rendering. The results demonstrate the capability of this filter for filling the holes and gaps as well as smoothing the contours of the lungs. The hole filling filter is better than morphological operations in edge preserving and smoothing.

\section{Experiment results}

The proposed method has been applied on 898 thorax CT scans. Here, 888 CT scans were freely downloaded from http: //grand-challenge.org/All_Challenges/, the others were provided by the Ninth People's Hospital of Shanghai Jiaotong University School of Medicine. In this paper, all 3D 16-bit

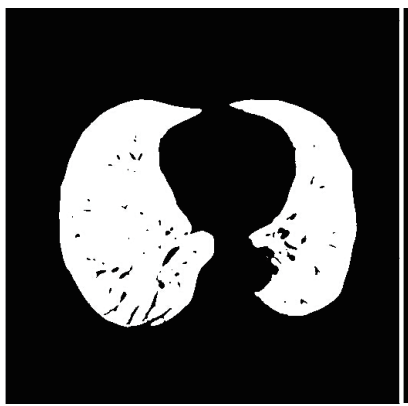

(a)

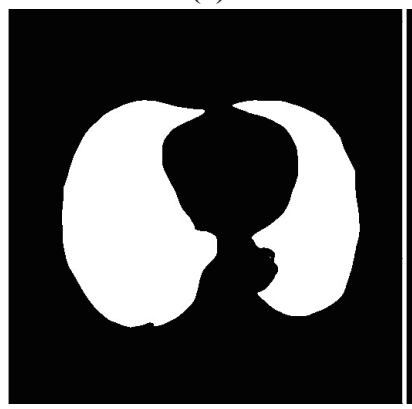

(c)

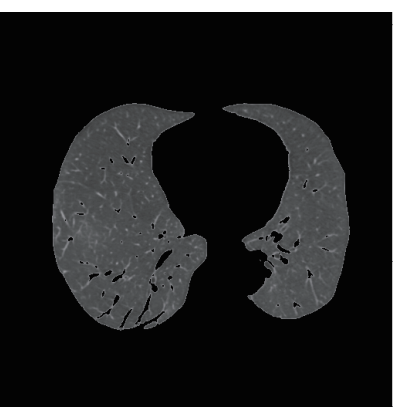

(b)

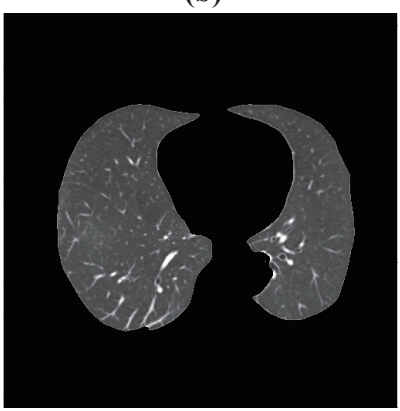

(d)
Figure 8: Examples for illustrating the hole filling filter. (a)-(b) The binary mask and segmented result before filling. (c)-(d) The binary mask and segmented result after filling.

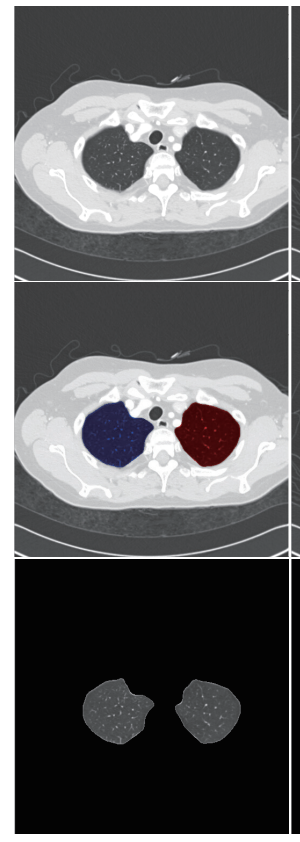

Slice:37

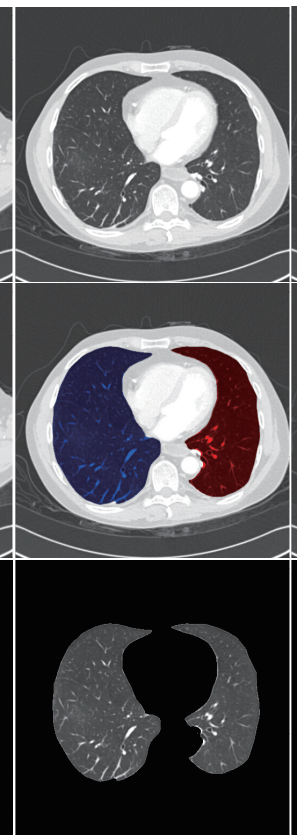

Slice: 164

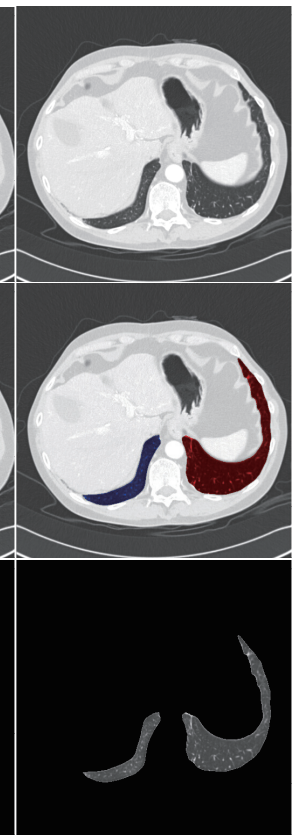

Slice:216
Figure 9: Segmented lungs by the proposed method. From top to bottom are 2D sectional slices, 2D sectional slices with different colors, segmented results, respectively.

images were converted into 3D 8-bit images by setting the window level at -600 and the window width at 1600 , according to the normal lung parenchyma (Hounsfield Units: $-700 \sim-400$, 


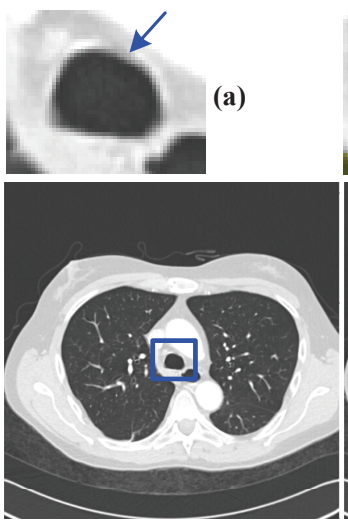

(f)

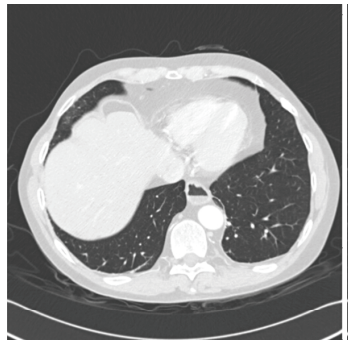

(k)

Original Image

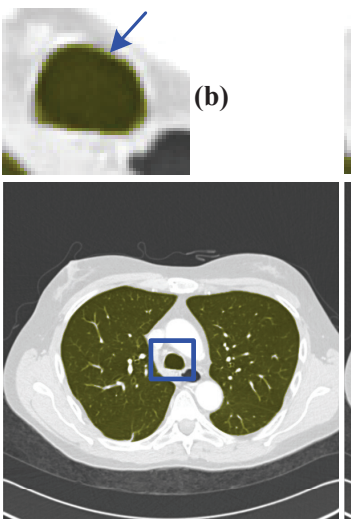

(g)

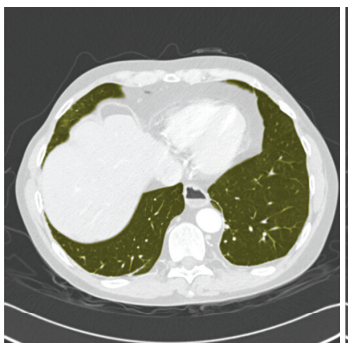

(I)

Threshold

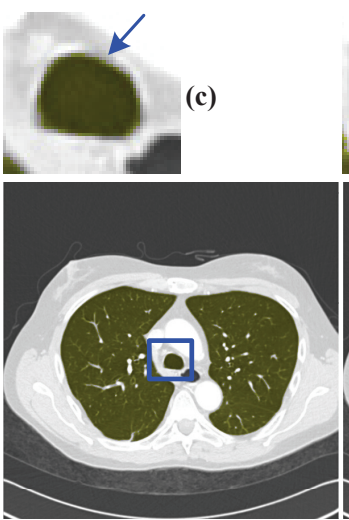

(h)

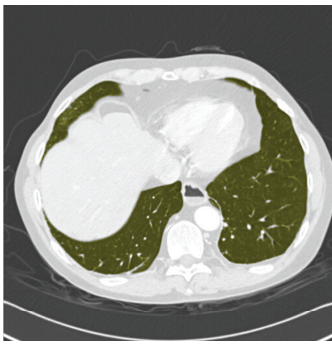

(m)

Region Growing

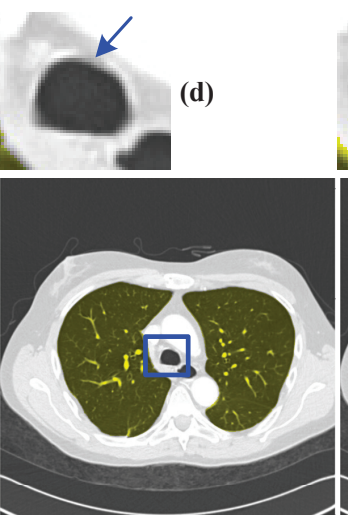

(i)

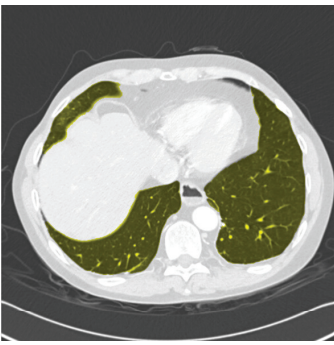

(n)

Graph Cuts

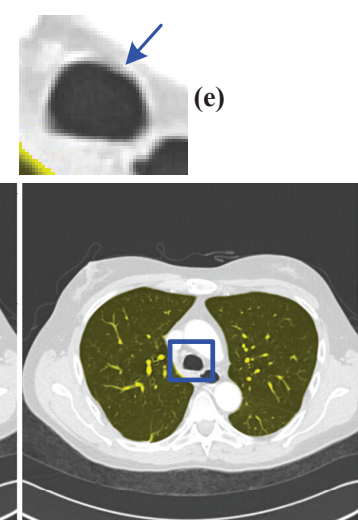

(j)

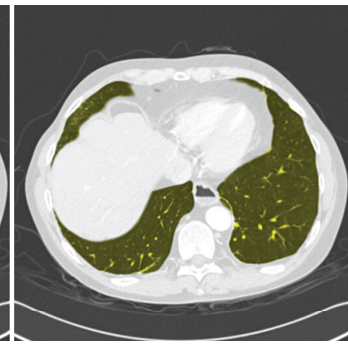

(o)

Our Method

Figure 10: Comparisons of different segmentation methods. The bronchus is surrounded by blue squares in (f)-(j) and then is amplified in (a)-(e). The area pointed by the arrow in (b) and (c) with pseudo-color indicates bronchus is not removed. The area with gray in (d) and (e) indicates the bronchus is removed.

mean $\approx-550$ ). The segmentation process is shown in Fig.9. The upper row represents the original images that are the frontend, intermediate, and rear-end layers of the chest CT image. The middle row represents the segmented left and right lungs with different colors. The lower row represents the segmented results without other structures. We can find that the segmented boundary is very similar to the human observation. All experiment results were run on a PC with Intel Core i7, 24G RAM and Nvidia GeForce GTX760. Here, the segmentation and visualization of the lungs on each chest CT image only takes about one minute.

\section{Discussions}

\subsection{Comparison with other methods}

In this section, we will present the segmentation results of our method compared to other methods. In Fig.10, three conventional methods (the threshold selection method, region growing method and graph cuts method) are compared with our method. Figs.10(f) and (k) represent the original CT images; Figs.10(g) and (l) are the segmented results of adaptive threshold selection method, and Figs.10(h) and (m) are the segmented results of region growing method. Obviously, the tracheas cannot be removed by these methods, and also some vascular structures are missed. On the contrast, as shown in Figs.10 (j) and (o), our method have similar performance to graph cuts method (Figs.10 (i) and (n)), and post-processing to remove the tracheas is not needed. In our framework, the left and right lungs are segmented separately because they belong to different connectivity sets, as shown in Fig.9 where the different colors represent different connectivity sets. The adaptive threshold and region growing methods can not segment the left and right lungs separately. Although the graph cuts based method has the ability to segment left and right lungs separately without post-processing, its efficiency is low compared to our method; see Section.4.2. Our method also provides visualization results compared to other methods.

\subsection{Quantitative evaluations}

To get an accurate evaluation of the proposed method, the criteria of dice similarity coefficient $(D S C)$, sensitivity $(S e)$, and specificity $(S p)$ are employed:

$$
\begin{aligned}
& D S C=\frac{2 T P}{2 T P+F P+F N} \\
& S e=\frac{T P}{T P+F N}, \quad S p=\frac{T N}{T N+F P}
\end{aligned}
$$

The $S e$ and $S p$ metrics are the ratio of well-classified lung and nonlung pixels, respectively. Where, $T P$ (true positives) is the total number of lung voxels which are correctly classified, $F N$ (false negatives) accounts for the number of lung voxels which are incorrectly classified, and FP (false positives) is the total number of those voxels that are incorrectly classified as lung voxels. Additionally, we also provide the $2 \mathrm{D}$ sectional 


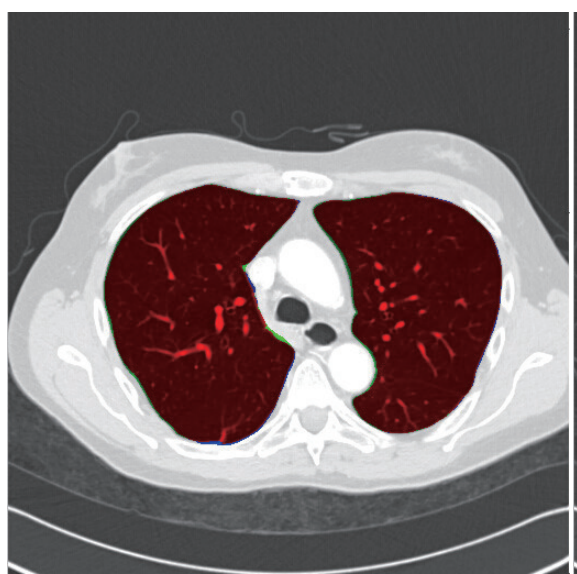

(a)

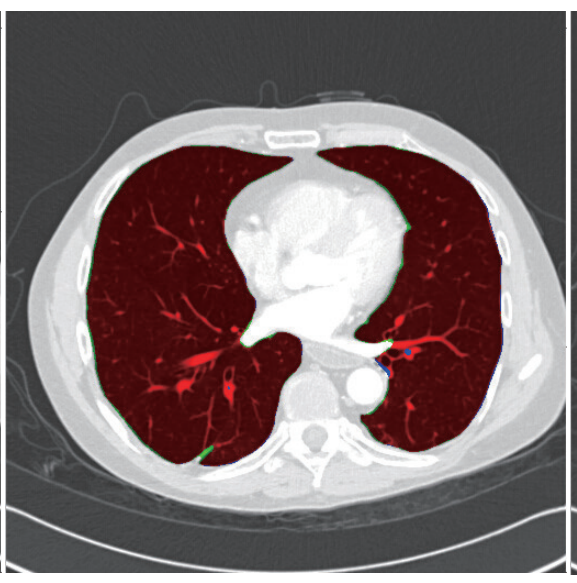

(b)

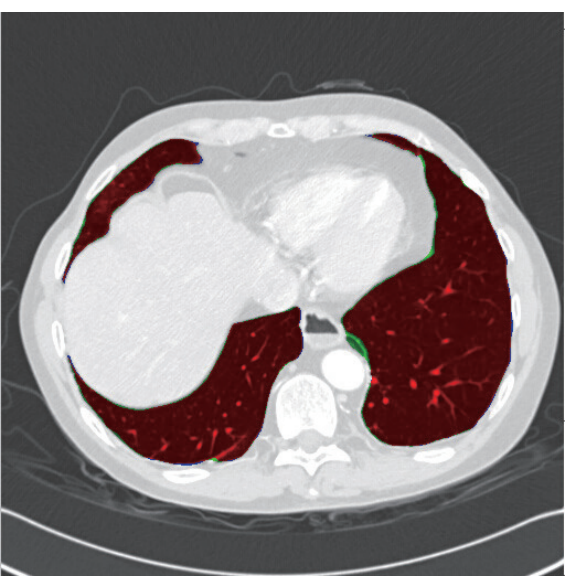

(c)

Figure 11: Comparison between the segmentation result and ground truth in (a), (b) and (c). The red regions represent the intersection of our segmentation results and manual segmentation results, the blue regions represent the false negative and the green represents the false positive.

slices comparison and time performance comparison. Fig.11 provides the comparison between the segmentation results and the ground truth. Both the blue and the green regions are very small, which represent that the missing segmentation rate (1$S e$ ) and false segmentation rate (1-Sp) are very low. In Table.1, we provide the accuracy comparison between our method and the graph cuts methods in $[19,22]$. We can find that the Dice similarity coefficient index of our method is higher than that of the method proposed in [19] and a litter lower than that of the method proposed in [22], but the computation time in our method is the best compared to the other two methods. Our method needs approximately 60 seconds to complete the lung segmentation and visualization, which is significantly less than in the other two methods. Thus, our framework can provide a clinically more acceptable processing time. Both $S e$ and $S p$ are almost equal to 1 , further demonstrating the effectiveness of the proposed technique in segmenting the lungs with pulmonary nodules.

Table 1: Time and accuracy comparison of different methods. DSC: Dice similarity coefficient. Se: true positive fractions. Sp: true negative fractions. Total time: computation time + interactions time. Time units: seconds

\begin{tabular}{lllll}
\hline Method & DSC & Se & Sp & Total time \\
\hline Ref.[19] & 0.974 & - & - & $>258$ \\
Ref.[22] & 0.987 & - & - & $>600$ \\
Ours & 0.981 & 0.961 & 0.997 & $\approx 60$ \\
\hline
\end{tabular}

In Table.2, we list the statistics of the time required for the different processing stages in our method. Here, both HCT and $C C T$ are less than 3 seconds providing a good guarantee for fast lung segmentation. The AIT of 20 seconds includes the time from the region selection of the $2 \mathrm{D}$ feature space to the completion of the lung segmentation and visualization. This interaction time is notably smaller than the 4.3 minutes of the method proposed in [19]. The HFT is approximately $35 \mathrm{sec}-$ onds for iteration 7 times, about five seconds per iteration on 260 slices. In summary, our approach provides a good guarantee in the outpatient service for clinicians, especially in face of more and more patients with pulmonology.

Table 2: Time of different operations. Time units: seconds. HCT: histogram computation time; CCT: connection computation time; HFT: hole filling time; AIT: all interactions time.

\begin{tabular}{lllll}
\hline HCT & CCT & HFT & AIT & Total time \\
\hline 2 & 3 & 35 & 20 & 60 \\
\hline
\end{tabular}

\subsection{Robustness analysis of $R 1$ selection in $2 D$ feature space}

The proposed method requires selecting a region $R 1$ to produce a primary segmentation of lungs, which is an intuitive strategy to attributes selection in 2D feature space. Generally, the appropriate region of lungs in 2D feature space is unknown. So the users can only roughly select a region for lungs in the $2 \mathrm{D}$ feature space. In Section 2.1 of this paper, the region $R 1$ of the lungs has been roughly determined in $2 \mathrm{D}$ feature space according to the attribute analysis. Now, we adjust the size of region $R 1$ to get different segmented results to evaluate the robustness of $R 1$ selection operation, as shown in Fig.12. Fig.12a shows 10 different sizes of region $R 1$, and then we can observe that their errors of $D S C, S e$ and $S p$ are within 0.015 compared with Table.1, see Fig.12b. In other words, the proposed method can get a satisfactory result no matter how the region $R 1$ is chosen in relatively feasible regions. Consequently, Fig. 12 further demonstrates the effectiveness of the proposed method in lung segmentation.

\subsection{User interface and interactions}

The user interface used in the method proposed in this paper is simple and intuitive. It consists mainly of four parts: the 


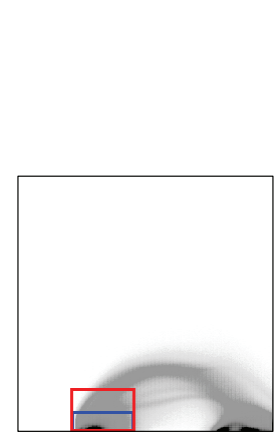

(a)

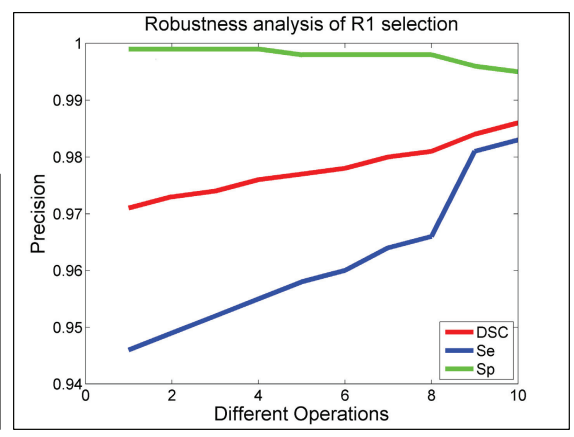

(b)
Figure 12: Robustness analysis of $R 1$ selection. (a) represents 10 regions $R 1$ of changing from small blue box to large red box gradually. (b) accuracy comparison for 10 levels of region $R 1$, which correspond to (a).

conventional user interface of the 2D feature space in Fig.13a; a pop-up context menu in Fig.13b; a 3D view in Fig.13c, and a sectional CT slice view in Fig.13d. Fig.13a is used for selecting and adjusting the region in the 2D feature space. Fig. $13 \mathrm{~b}$ will pop up by right-clicking, and is used for selecting the seed, computing the connected sets from $\Phi_{U}$ and filling the holes and gaps. Fig. $13 \mathrm{c}$ is for displaying and operating the volume rendering results. Fig. $13 \mathrm{~d}$ is used for displaying the original sectional CT slices or the segmented sectional CT slices with pseudo-color.

Based on the user interface and the method presented in this paper, the user interactions for segmenting and visualizing the lungs in $\Phi_{U}$ may become simple and more intuitive. As an example of the steps of the process, we firstly select a region in the 2D feature space, as shown in Fig.5a, and visualize all voxels of $\Phi_{U}$ in volume rendering, as shown in Fig.5b. By right-clicking the lung in volume rendering result, and selecting the "SelectedSeeds" item in the pop-up context menu of Fig. 13b, a seed will be marked on that position, as shown in Fig.6a. Then, by right-clicking the lung and selecting the "Connectivity" item in the pop-up context menu, the connected set of the lungs can be automatically extracted from $\Phi_{U}$, as shown in Fig.6c. Alternatively, if we select two seed points on the left and right bronchus, as shown in Fig.6b, the left and right lungs can be individually extracted and visualized with different colors, as shown in Fig.6d. Then, we can right click the lung, select the "FillingFilter" item in the pop-up context menu, and automatically repair the missing small vascular structures and pulmonary nodules. Finally, when the lung is separated from the chest CT images, we can open the $2 \mathrm{D}$ view of the Fig. $13 \mathrm{~d}$ to see the original sectional CT slices or the segmented sectional CT slices, according to the color mode.

In additional, the lungs and pulmonary nodules can be visualized simultaneously with different colors and opacities in volume rendering by adjusting the "Label" item and "Opacity" item in Fig.13a. Here, the "Label" item and "Opacity" item is one-to-one correspondence, different labels represent different structures, such lungs and pulmonary nodules.

As has been demonstrated, the user interface and the interactive mode are intuitive and simple in this CAD system. This

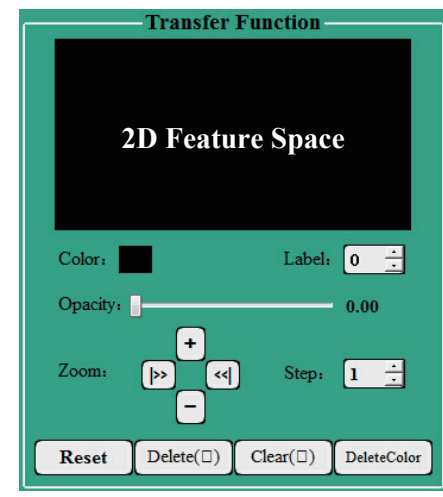

(a)

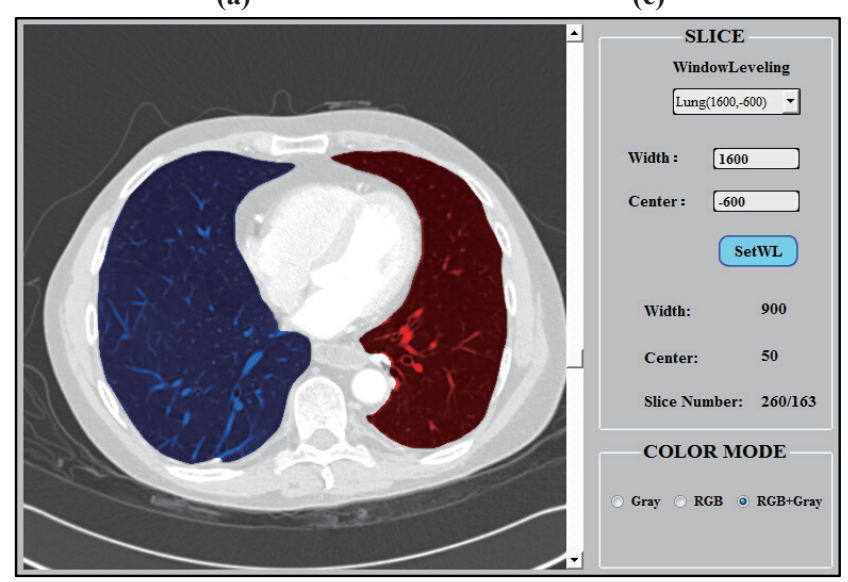

(d)
Figure 13: The User interface of our framework. (a) The conventional user interface of the 2D feature space. (b) A pop-up context menu. (c) A 3D view for displaying and operating visual results. (d) A 2D view for displaying 2D sectional slices.

enables the clinicians to segment and visualize the lungs and pulmonary nodules easily from the chest CT images. Moreover, the processing time is more acceptable for the clinicians in outpatient service.

\subsection{Comments on CT images from different imaging systems}

In this paper, we propose a visually guided framework for lung segmentation and visualization in chest CT images. This framework is robust to CT images from different imaging systems, such as plain CT image (e.g., Fig.7) and enhanced CT image (e.g., Figs.6, 9, 10, and 11). Due to this framework first segments the pulmonary tissue and then repairs the missing vascular structures, slight difference of CT images acquired from different imaging systems does not affect the robustness. Hence, this framework can be also applied in dual-energy CT image (DECT)[28, 29, 30, 31]. In conclusion, this framework is a robust tool in preoperative planning.

\section{Conclusions}

The graph cuts method is currently the latest and most intuitive lung segmentation method. However, the time consumption seriously limits its application for the clinicians in outpa- 
tient service. We propose a visually guided framework to segment and visualize the lungs and pulmonary nodules by combining 2D feature space and spatial connectivity computation, while avoiding the excessive time-consumption of the graph cuts methods. As a result, this method can segment the left and right lungs separately without post-processing. The lungs with pulmonary nodules can be visualized simultaneously in volume rendering, which provides a intuitive information for the clinicians in outpatient service. In addition, we have asked some clinicians to evaluate the usability by using the framework. Experimental results also demonstrate the effectiveness of the proposed method, which significantly improves the work efficiency of the clinicians and reduce the dependence on the radiologists for lung segmentation and visualization in outpatient service, especially in face of more and more patients with pulmonology.

\section{Acknowledgements}

This work was supported in part by the 973 program of China (No.2013CB329401), NSFC of China (No.61375020, 61572317) and Cross Research Fund of Biomedical Engineering of SJTU (No.YG2016MS55).

\section{References}

[1] Y. J. Lee, M. Lee, N. Kim, J. B. Seo, J. Y. Park, Automatic left and right lung separation using free-formed surface fitting on volumetric CT, Journal of Digital Imaging. 27(4), pp.538-547 (2014)

[2] A. Karthike]yan, M. Vallianmmai, Lungs segmentation using multi-level thresholding in CT images, Int. J. Electron. Comput. Sci. Eng. 1(3), pp.1509-1513 (2012).

[3] S. Hu, E.A. Hoffman, J.M. Reinhardt, Automatic lung segmentation for accurate quantitation of volumetric X-ray CT images, IEEE Trans. Medical Imaging. 20(6), pp.490-498 (2001).

[4] L. Tseng, L. Huang. An adaptive thresholding method for automatic lung segmentation in CT images. In:IEEE AFRICON, (2009) September 2325; Nairobi, Kenya

[5] J. Pu, J. Roos, C.A. Yi, S. Napel, G.D. Rubin, D.S. Paik, Adaptive border marching algorithm: automatic lung segmentation on chest CT images, Computerized Medical Imaging and Graphics. 32, pp.452-462 (2008).

[6] S. Sun, C. Bauer, R. Beichel, Automated 3-D Segmentation of Lungs with lung cancer in CT Data using a novel robust active shape model approach, IEEE Trans. Medical Imaging. 31(2), pp.449-460 (2012).

[7] N.M. Noor, J.C.M. Than, O.M. Rijal, R.M. Kassim, A. Yunus, A.A. Zeki, M. Anzidei, L. Saba, J.S. Suri, Automatic Lung Segmentation Using Control Feedback System: Morphology and Texture Paradigm, Journal of medical systems. 39(3), pp.1-18 (2015).

[8] A. Mansoor, U. Bagci, Z. Xu, B. Foster, K.N. Olivier, J.M. Elinoff, A.F. Suffredini, J.K. Udupa, A generic approach to pathological lung segmentation, IEEE Trans. Medical Imaging. 33(12), pp.2293-2310 (2014).

[9] I. Sluimer, M. Prokop, B. van Ginneken, Toward automated segmentation of the pathological lung in CT, IEEE Trans. Med. Imaging. 24(8), pp.1025-1038 (2005)

[10] M.J. Gangeh, L. Sorensen, S.B. Shaker, M.S. Kamel, M. Bruijne, M. Loog. A texton-based approach for the classification of lung parenchyma in CT images. In:Medical Image Computing and Computer-Assisted Intervention-MICCAI, (2010) September 20-24; Beijing, China

[11] J. Yao, A. Dwyer, R.M. Summers, D.J. Mollura, Computeraided diagnosis of pulmonary infections using texture analysis and support vector machine classification, Academic radiology. 18(3), pp.306-314 (2011).

[12] M. Ceylan, Y. ZBAY, O. N. UAN, and E. Yildirim, A novel method for lung segmentation on chest CT images:complex-valued artificial neural network with complex wavelet transform, Turkish Journal of Electrical Engineering and Computer Sciences. 18(4), pp.613-624 (2010).
[13] N. Mesanovic, M. Grgic, H. Huseinagic, M. Males, E. Skejic, M. Smajlovic. Automatic CT image segmentation of the lungs with region growing algorithm. In:18th International Conference on Systems, Signals and Image Processing-IWSSIP, (2011) June 16-18; Sarajevo, Bosnia and Herzegovina

[14] R. Pohle, K.D. Toennies, Segmentation of medical images using adaptive region growing, Medical Imaging 2000. International Society for Optics and Photonics. 4322, pp.1337-1346 (2001).

[15] J. Dehmeshki, H. Amin, M. Valdivieso, X. Ye, Segmentation of pulmonary nodules in Thoracic CT scans: A region growing approach, IEEE Trans. Medical Imaging. 27(4), pp.467-480 (2008).

[16] L.w. Hedlund, R.F. Anderson, P.L. Goulding, J.W. Beck, E.L. Effmann, and C.E. Putman, Two mehods for isolating the lung area of a CT scan for density information, Radiology. 144, pp.353-357 (1982).

[17] W.A. Kalender, H. Fichte, W. Bautz, and M. Skalej, Semiautomatic evaluation procedures for quantitative CT of the lung, Journal of Computer Assisted Tomography. 15(2), pp.248-255 (1991).

[18] Y. Boykov, O. Veksler, R. Zabih, Fast Approximate Energy Minization via graph cuts, IEEE Trans. Pattern Analysis and Machine Intelligence. 23(11), pp.1222-1239 (2001).

[19] S. Sun, M. Sonka, R. Beichel. Graph-based 4D lung segmentation in CT images with expert-guided computer-aided refinement. In:IEEE 10th International Symposium on Biomedical Imaging, (2013) April 7-11; San Francisco, USA

[20] K. Nakagomi, A. Shimizu, H. Kobatake, M. Yakami, K. Fujimoto, K. Togashi, Multi-Shape cuts with neigbor prior constrains and its application to lung segmentation from a chest CT volume, Medical Image Analysis. 17(1), pp.62-77 (2013).

[21] A. M. Ali, A. A. Farag. Automatic lung segmentation of volumetric lowdose CT scans using graph cuts. In: International Symposium on Visual Computing, (2008) December 1-3; Las Vegas, USA

[22] S. Dai, K. Lu, J. Dong, A novel approach of lung segmentation on chest CT images using graph cuts, Neurocomputing. 168, pp.799-807 (2015).

[23] H. Ning, R. Yang, A. Ma, X. Wu, Interactive 3D medical data cutting using closed curve with arbitrary shape, Computerized Medical Imaging and Graphics. 40, pp.120-127 (2015).

[24] J. Kniss, G. Kindlmann, C. Hansen, Multidimensional transfer functions for interactive volume rendering, IEEE Trans. Visualization and Computer Graphics. 8(3), pp.270-285 (2002).

[25] W. Schroeder, L. Ng, J. Cates, The ITK Software Guide Second Edition Updated for ITK version 2.4, Vol.525, FEBS Letters (2005).

[26] H. Pfister, B. Lorensen, C. Bajaj, G. Kindlmann, W. Schroeder, L. S. Avila, K. Martin, R. Machiraju, and J. Lee, The transfer function bakeoff, IEEE Computer Graphics and Applications. 21(3), pp.16-22 (2001).

[27] P. Ljung, J. Krger, E. Groller, M. Hadwiger, C. D. Hansen, A. Ynnerman, State of the Art in Transfer Functions for Direct Volume Rendering, Computer Graphics Forum. 35(3), pp.669-691 (2016).

[28] K. L. Goh, S. C. Liew, B. H. Hasegawa, Energy-dependent systematic errors in dual-energy X-ray CT, IEEE Trans. Nuclear Science. 44(2), pp.212-217 (1997).

[29] K. L. Goh, S. C. Liew, B. H. Hasegawa, Correction of energy-dependent systematic errors in dual-energy X-ray CT using a basis material coefficients transformation method, IEEE Trans. Nuclear Science. 44(6), pp.2419-2424 (1997).

[30] I. Vlahos, R. Chung, A. Nair, R. Morgan, Dual-energy CT: vascular applications, American Journal of Roentgenology. 199(5_supplement), pp.S87-S97 (2012).

[31] R. Vliegenthart, G. J. Pelgrim, U. Ebersberger, G. W. Rowe, M. Oudkerk, U. J. Schoepf, Dual-energy CT of the heart, American Journal of Roentgenology. 199(5_supplement), pp.S54-S63 (2012). 